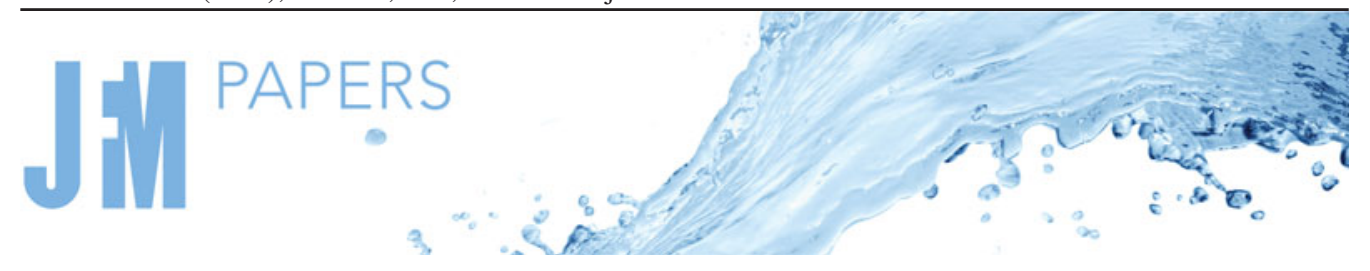

\title{
Wind-induced changes to surface gravity wave shape in shallow water
}

\author{
Thomas Zdyrski ${ }^{1, \dagger}$ and Falk Feddersen ${ }^{1}$ \\ ${ }^{1}$ Scripps Institution of Oceanography, UCSD, La Jolla, CA 92092-0209, USA
}

(Received 22 September 2020; revised 4 December 2020; accepted 1 January 2021)

\begin{abstract}
Wave shape (e.g. wave skewness and asymmetry) impacts sediment transport, remote sensing and ship safety. Previous work showed that wind affects wave shape in intermediate and deep water. Here, we investigate the effect of wind on wave shape in shallow water through a wind-induced surface pressure for different wind speeds and directions to provide the first theoretical description of wind-induced shape changes. A multiple-scale analysis of long waves propagating over a shallow, flat bottom and forced by a Jeffreys-type surface pressure yields a forward or backward Korteweg-de Vries (KdV)-Burgers equation for the wave profile, depending on the wind direction. The evolution of a symmetric, solitary-wave initial condition is calculated numerically. The resulting wave grows (decays) for onshore (offshore) wind and becomes asymmetric, with the rear face showing the largest shape changes. The wave profile's deviation from a reference solitary wave is primarily a bound wave and trailing, dispersive, decaying tail. The onshore wind increases the wave's energy and skewness with time while decreasing the wave's asymmetry, with the opposite holding for offshore wind. The corresponding wind speeds are shown to be physically realistic, and the shape changes are explained as slow growth followed by rapid evolution according to the unforced $\mathrm{KdV}$ equation.
\end{abstract}

Key words: surface gravity waves, wind-wave interactions, air/sea interactions

\section{Introduction}

The study of wind and ocean wave interactions began with Jeffreys (1925) and continues to be an active field of research (e.g. Janssen 1991; Donelan et al. 2006; Sullivan \& McWilliams 2010). Many theoretical studies (e.g. Jeffreys 1925; Miles 1957; Phillips 1957) focus on calculating wind-induced growth rates and often employ phase-averaging techniques. However, experimental (e.g. Leykin et al. 1995; Feddersen \& Veron 2005) and

$†$ Email address for correspondence: tzdyrski@uscd.edu

(C) The Author(s), 2021. Published by Cambridge University Press. This is an Open Access article, distributed under the terms of the Creative Commons Attribution licence (http://creativecommons.org/ licenses/by/4.0/), which permits unrestricted re-use, distribution, and reproduction in any medium, provided the original work is properly cited. 


\section{T. Zdyrski and F. Feddersen}

theoretical (e.g. Zdyrski \& Feddersen 2020) studies have shown wind can also influence wave shape, quantified by third-order shape statistics such as skewness and asymmetry, corresponding to vertical and horizontal asymmetry, respectively. Furthermore, while many numerical studies on coupled wind and waves employ sinusoidal water waves and therefore neglect wind-induced shape changes (e.g. Hara \& Sullivan 2015; Husain et al. 2019), some recent numerical studies have incorporated wind-induced changes to the wave field using coupled air-water simulations (e.g. Liu et al. 2010; Hao \& Shen 2019) or direct numerical simulations of two-fluid flows (e.g. Zonta, Soldati \& Onorato 2015; Yang, Deng \& Shen 2018). Wave shape influences sediment transport, affecting beach morphodynamics (e.g. Drake \& Calantoni 2001; Hoefel \& Elgar 2003), while wave skewness affects radar altimetry signals (e.g. Hayne 1980) and asymmetry influences ship responses to wave impacts (e.g. Soares, Fonseca \& Pascoal 2008).

Waves in shallow water, where $k h \ll 1$ (with $h$ the water depth, $k=2 \pi / \lambda$ the wavenumber and $\lambda$ the wavelength), differ qualitatively from those in intermediate $(k h \sim$ 1) to deep $(k h \gg 1)$ water. For waves with small amplitudes $a_{0} \ll h$, leveraging the small parameters $a_{0} / h \sim(k h)^{2} \ll 1$ yields the Boussinesq equations with weak dispersion and nonlinearity. When dispersion balances nonlinear focusing, a special class of waves, known as solitary waves, are formed and appear in environments ranging from nonlinear optical pulses (e.g. Kivshar 1993) to astrophysical dusty plasmas (e.g. Sahu \& Tribeche 2012). These well-understood waves are often used to study fluid dynamical (e.g. Munk 1949; Hammack \& Segur 1974; Miles 1979; Lin \& Liu 1998) and engineering (e.g. Monaghan \& Kos 1999; Lin 2004; Xu, Chen \& Chen 2018) contexts owing to their simplicity. One of the simplest equations displaying solitary waves is the Korteweg-de Vries (KdV) equation, which incorporates dispersion and nonlinearity. When augmented with a dissipative term, this becomes the $\mathrm{KdV}$-Burgers equation, with applications to damped internal tides (e.g. Sandstrom \& Oakey 1995), electron waves in graphene (e.g. Zdyrski \& McGreevy 2019) and viscous flow in blood vessels (e.g. Antar \& Demiray 1999). While field observations (e.g. Cavaleri \& Rizzoli 1981) have investigated the wind-induced growth of shallow-water waves, the interaction of wind and shallow-water waves has not yet been formulated into a simple equation such as the $\mathrm{KdV}$-Burgers equation.

The influence of wind on wave shape has been previously investigated in intermediate and deep water (Zdyrski \& Feddersen 2020). However, the coupling between wind and wave shape has not yet been investigated in shallow water. To investigate wind and surface wave interactions in shallow water over a flat bottom, we introduce a wind-induced pressure term to the Boussinesq equations in $\S 2$. The resulting $\mathrm{KdV}-$ Burgers equation governs a solitary wave's evolution, which we solve numerically to yield the wave's energy, skewness and asymmetry in $\S 3$. We calculate the wind speed, discuss the asymmetry and compare our results to intermediate- and deep-water waves in $\S 4$.

\section{Derivation of the $\mathrm{KdV}-$ Burgers equation}

\subsection{Governing equations}

We treat the flow as irrotational and inviscid and neglect surface tension. Furthermore, we restrict ourselves to planar wave propagation in the $+x$ direction. Finally, we choose a coordinate system with $z=0$ at the mean water level and a horizontal, flat bottom located at $z=-h$. Then, the incompressibility condition and standard boundary conditions are

$$
\begin{gathered}
0=\phi_{x x}+\phi_{z z} \quad \text { on }-h<z<\eta, \\
0=\phi_{z} \quad \text { on } z=-h,
\end{gathered}
$$


Wind-induced wave shape changes in shallow water

$$
\begin{gathered}
\phi_{z}=\eta_{t}+\phi_{x} \eta_{x} \quad \text { on } z=\eta \\
0=\frac{p}{\rho_{w}}+g \eta+\phi_{t}+\frac{1}{2}\left[\phi_{x}^{2}+\phi_{z}^{2}\right] \quad \text { on } z=\eta .
\end{gathered}
$$

Here, $\eta(x, t)$ is the wave profile, $\phi(x, z, t)$ is the flow's velocity potential related to the velocity $\boldsymbol{u}=\boldsymbol{\phi}, p(x, t)$ is the surface pressure, $g$ is the gravitational acceleration and $\rho_{w}$ is the water density. We used the $\phi$ gauge freedom to absorb the Bernoulli 'constant' $C(t)$ in the dynamic boundary condition. We seek a solitary, progressive wave which decays at infinity, $\eta(\boldsymbol{x}, t) \rightarrow 0$ as $|\boldsymbol{x}| \rightarrow \infty$, with similar conditions on $\boldsymbol{u}$. We choose a coordinate system where the average bottom horizontal velocity vanishes,

$$
\overline{\frac{\partial \phi}{\partial x}}=0 \quad \text { on } z=-h
$$

with the overline a spatial average $\bar{f}:=\lim _{L \rightarrow \infty} \int_{-L}^{L} f \mathrm{~d} x /(2 L)$. Additionally, we assume the surface pressure $p(x, t)$ is a Jeffreys-type forcing (Jeffreys 1925),

$$
p(x, t)=P \frac{\partial \eta(x, t)}{\partial x} .
$$

Here, $P$ is proportional to $(U-c)^{2}$, with $c$ the wave's nonlinear phase speed and $U$ the wind speed (cf. $\S 4.1$ ). Note that $P>0$ corresponds to ('onshore') wind in the same direction as the wave while $P<0$ denotes ('offshore') wind opposite the wave. We use a Jeffreys forcing for its analytic simplicity and clear demonstration of wind-wave coupling. Jeffrey's separated sheltering mechanism is likely only relevant in special situations (e.g. near breaking, Banner \& Melville 1976, or for steep waves under strong winds, Touboul \& Kharif 2006; Tian \& Choi 2013). Additionally, numerical simulations of sinusoidal waves suggest the peak surface pressure is shifted approximately $135^{\circ}$ from the wave peak, while Jeffreys would give a $90^{\circ}$ shift (Husain et al. 2019). However, a fully dynamic coupling between wind and waves - necessary for an accurate surface pressure over a non-sinusoidal, dynamic water surface - is outside the scope of this paper. Furthermore, the applicability of Jeffreys forcing to extreme waves means our theory could apply to the wind forcing of rogue waves in shallow water (Kharif et al. 2008).

\subsection{Non-dimensionalization}

We non-dimensionalize our system with the known characteristic scales: the horizontal length scale $L$ over which $\eta$ changes rapidly, expressed as an effective wavenumber $k_{E}:=$ $2 \pi / L$; the (initial) wave amplitude $a_{0}=H_{0} / 2$ (i.e. half the wave height $H_{0}$ ); the depth $h$; the gravitational acceleration $g$; and the wind speed $U$, expressed as a pressure magnitude $P \propto \rho_{a}(U-c)^{2}$, with $\rho_{a} \approx 1.225 \times 10^{-3} \rho_{w}$ the density of air. Denoting non-dimensional variables with primes, we have

$$
\left.\begin{array}{c}
x=\frac{x^{\prime}}{k_{E}}=h \frac{x^{\prime}}{\sqrt{\mu_{E}}}, \quad t=\frac{t^{\prime}}{k_{E} c_{0}}=\frac{t^{\prime}}{\sqrt{\mu_{E}}} \sqrt{\frac{h}{g}}, \quad \eta=a_{0} \eta^{\prime}=h \varepsilon \eta^{\prime}, \\
z=h z^{\prime}, \quad P=\varepsilon P^{\prime} \frac{\rho_{w} g}{k_{E}}=\frac{\varepsilon}{\sqrt{\mu_{E}}} P^{\prime} \rho_{w} c_{0}^{2}, \quad \phi=\phi^{\prime} \frac{a_{0}}{k_{E}} \sqrt{\frac{g}{h}}=\frac{\phi^{\prime} \varepsilon}{\sqrt{\mu_{E}}} c_{0} h,
\end{array}\right\}
$$

with linear, shallow-water phase speed $c_{0}=\sqrt{g h}$. Our system's dynamics is controlled by three small, non-dimensional parameters: $\varepsilon:=a_{0} / h, \mu_{E}:=\left(k_{E} h\right)^{2}$ and $P k_{E} /\left(\rho_{w} g\right)$. 
We will later require $O(\varepsilon)=O\left(\mu_{E}\right)=O\left(P k_{E} /\left(\rho_{w} g\right)\right)$. Now, our non-dimensional equations take the form

$$
\begin{gathered}
0=\mu_{E} \phi_{x^{\prime} x^{\prime}}^{\prime}+\phi_{z^{\prime} z^{\prime}}^{\prime} \quad \text { on }-1<z^{\prime}<\varepsilon \eta^{\prime} \\
0=\phi_{z^{\prime}}^{\prime} \quad \text { on } z^{\prime}=-1, \\
\phi_{z^{\prime}}^{\prime}=\mu_{E} \eta_{t^{\prime}}^{\prime}+\varepsilon \mu_{E} \phi_{x^{\prime}}^{\prime} \eta_{x^{\prime}}^{\prime} \quad \text { on } z^{\prime}=\varepsilon \eta^{\prime}, \\
0=\varepsilon P^{\prime} \eta_{x^{\prime}}^{\prime}+\eta^{\prime}+\phi_{t^{\prime}}^{\prime}+\frac{1}{2}\left(\varepsilon \phi_{x^{\prime}}^{\prime 2}+\frac{\varepsilon}{\mu_{E}} \phi_{z^{\prime}}^{\prime 2}\right) \quad \text { on } z^{\prime}=\varepsilon \eta^{\prime} .
\end{gathered}
$$

We will drop the primes throughout the remainder of this section for readability.

\subsection{Boussinesq equations, multiple-scale expansion, $K d V$ equation and initial condition}

Here, we modify the Boussinesq equation's derivation provided by Mei, Stiassnie \& Yue (2005) or Ablowitz (2011) by including the surface pressure forcing in (2.4). Taylor expanding the velocity potential $\phi$ about the bottom, $z=-1$, and applying Laplace's equation (2.8) and the bottom boundary condition (2.9) yields an expansion of $\phi$ in terms of $\mu_{E} \ll 1$ and the velocity potential at the bottom, $\varphi:=\left.\phi\right|_{z=-1}$. This $\phi$ expansion can be substituted into the two remaining boundary equations, (2.10) and (2.11), to give the Boussinesq equations with a pressure forcing term,

$$
\begin{gathered}
\partial_{t} \eta+\partial_{x}^{2} \varphi+\varepsilon \partial_{x}\left(\eta \partial_{x} \varphi\right)-\frac{1}{6} \mu_{E} \partial_{x}^{4} \varphi=O\left(\mu_{E}^{2}\right), \\
\partial_{t} \varphi+\varepsilon P \partial_{x} \eta+\eta-\frac{1}{2} \mu_{E} \partial_{t} \partial_{x}^{2} \varphi+\frac{1}{2} \varepsilon\left(\partial_{x} \varphi\right)^{2}=O\left(\mu_{E}^{2}\right) .
\end{gathered}
$$

Further, we will now assume $O(\varepsilon)=O\left(\mu_{E}\right) \ll 1$.

We now expand $t$ using multiple time scales $t_{n}=\varepsilon^{n} t$ for $n=0,1$, so all time derivatives become $\partial_{t} \rightarrow \partial_{t_{0}}+\varepsilon \partial_{t_{1}}$. Then, we write $\eta$ and $\varphi$ as asymptotic series of $\varepsilon$,

$$
\eta(x, t)=\sum_{k=0}^{\infty} \varepsilon^{k} \eta_{k}\left(x, t_{0}, t_{1}\right) \quad \text { and } \quad \varphi(x, t)=\sum_{k=0}^{\infty} \varepsilon^{k} \varphi_{k}\left(x, t_{0}, t_{1},\right) .
$$

Now, we will reduce the Boussinesq equations, (2.12) and (2.13), to the $\mathrm{KdV}$ equation following a similar method to Mei et al. (2005) and Ablowitz (2011). Collecting order-one terms $O\left(\varepsilon^{0}\right)$ from (2.12) and (2.13) gives a wave equation for $\eta_{0}$ and $\phi_{0}$. The right-moving solutions are

$$
\varphi_{0}=f_{0}\left(x-t_{0}, t_{1}\right) \quad \text { and } \quad \eta_{0}=f_{0}^{\prime}\left(x-t_{0}, 1\right) \quad \text { with } f_{0}^{\prime}:=\left.\frac{\partial f_{0}\left(\theta, t_{1}\right)}{\partial \theta}\right|_{\theta=x-t_{0}} .
$$

Continuing to the next order of perturbation theory, we retain terms of $O(\varepsilon)$,

$$
\begin{gathered}
\frac{\partial \eta_{1}}{\partial t_{0}}+\frac{\partial^{2} \varphi_{1}}{\partial x^{2}}=-\frac{\partial \eta_{0}}{\partial t_{1}}-\frac{\partial}{\partial x}\left(\eta_{0} \frac{\partial \varphi_{0}}{\partial x}\right)+\frac{1}{6} \frac{\mu_{E}}{\varepsilon} \frac{\partial^{4} \varphi_{0}}{\partial x^{4}} \\
\eta_{1}+\frac{\partial \varphi_{1}}{\partial t_{0}}=-P \frac{\partial \eta_{0}}{\partial x}-\frac{\partial \varphi_{0}}{\partial t_{1}}+\frac{1}{2} \frac{\mu_{E}}{\varepsilon} \frac{\partial^{3} \varphi_{0}}{\partial t_{0} \partial^{2} x}-\frac{1}{2}\left(\frac{\partial \varphi_{0}}{\partial x}\right)^{2} .
\end{gathered}
$$


Inserting our leading-order solutions for $\eta_{0}$ and $\varphi_{0}$, eliminating $\eta_{1}$ and preventing resonant forcing of $\varphi_{1}$ gives the $\mathrm{KdV}$-Burgers equation,

$$
\frac{\partial \eta_{0}}{\partial t_{1}}+\frac{3}{2} \eta_{0} \frac{\partial \eta_{0}}{\partial x}+\frac{1}{6} \frac{\mu_{E}}{\varepsilon} \frac{\partial^{3} \eta_{0}}{\partial x^{3}}=-P \frac{1}{2} \frac{\partial^{2} \eta_{0}}{\partial x^{2}} .
$$

Note that (2.18) has a rescaling symmetry, with $\mu_{E} \rightarrow \lambda^{2} \mu_{E}$ equivalent to taking $\left(x, t_{0}, t_{1}, P\right) \rightarrow\left(x, t_{0}, t_{1}, P\right) / \lambda$. Therefore, we fix the length scale (equivalently, $\left.k_{E}\right)$ by choosing $\mu_{E}=6 \varepsilon$. Note that incorporating slowly varying bottom bathymetry $\partial_{x} h=O(\varepsilon)$ can yield an equation of the form (2.18) with spatially varying coefficients (e.g. Johnson 1972; Ono 1972), although such an analysis is outside the scope of this study.

For offshore wind, the pressure term $P \partial_{x}^{2} \eta_{0}$ acts as a positive viscosity causing damping, and (2.18) is the (forward) $\mathrm{KdV}-$ Burgers equation with $P<0$. However, for onshore wind, the viscosity is negative and causes wave growth, yielding the backward KdV-Burgers equation with $P>0$. The backward $\mathrm{KdV}$-Burgers equation is ill posed in the sense of Hadamard because the solution is highly sensitive to changes in the initial condition (Hadamard 1902). While a finite-time singularity (i.e. wave breaking) is likely, the multiple-scale expansion used to derive (2.18) is only valid for time intervals of $O(1 / \varepsilon)$, and we limit our analysis to short times removing the need to regularize the solution.

The solitary-wave solutions of the unforced $(P=0) \mathrm{KdV}$ equation exist due to a balance of dispersion $\partial_{x}^{3} \eta_{0}$ with focusing nonlinearity $\eta_{0} \partial_{x} \eta_{0}$ and have the form (e.g. Mei et al. 2005)

$$
\eta_{0}=H_{0} \operatorname{sech}^{2}\left(\frac{x}{\Delta}\right) \quad \text { with } \Delta=\sqrt{\frac{8}{H_{0}}},
$$

in a co-moving frame with $H_{0}>0$ an order-one parameter. For reference, unforced solitary waves travel relative to the laboratory frame with non-dimensional, nonlinear phase speed (e.g. Mei et al. 2005)

$$
c=1+\varepsilon \frac{H_{0}}{2} .
$$

We use (2.19) for our initial condition and choose $H_{0}=2$ so the initial, dimensional amplitude $a_{0}$ is half the wave height (cf. $\S 2.2$ ). Note that the unforced KdV equation also has periodic solutions known as cnoidal waves. For a fixed height, these cnoidal waves have a smaller characteristic wavelength $1 / k_{E}$ than solitary waves and can be studied by choosing larger $\mu_{E}>6 \varepsilon$ (cf. $\S 4.3$ ). However, wind-induced shape changes are more readily understood when considering solitary waves owing to their reduced number of free parameters (i.e. $\mu_{E}$ ). Furthermore, since solitary waves are well understood and highly relevant to fluid dynamical systems (e.g. Hammack \& Segur 1974; Miles 1979; Lin \& Liu 1998; Monaghan \& Kos 1999), we will restrict our analysis to solitary waves for brevity and clarity. The wind-forcing term $P \partial_{x}^{2} \eta_{0}$ in (2.18) disrupts the solitary wave's balance of dispersion and nonlinearity, inducing growth/decay and shape changes. The KdV-Burgers equation has no known solitary-wave solutions, so we will solve it numerically.

\subsection{Numerics and shape statistics}

To solve (2.18) numerically, we will use the Dedalus spectral solver (Burns et al. 2020) which implements a generalized tau method with a Chebyshev basis. Since the onshore wind, $P>0$ case is ill posed, we require an implicit solver, so time stepping is done with coupled four-stage, third-order diagonally implicit Runge-Kutta and explicit 


\section{T. Zdyrski and F. Feddersen}

Runge-Kutta schemes. The spatial domain has a length of $L=80$, and we require $\eta_{0}=0$ at $x^{\prime}=-40$ and $\eta_{0}=\partial_{x} \eta_{0}=0$ at $x^{\prime}=40$. We employ $N_{c}=1600$ Chebyshev coefficients and zero padding with a scaling factor of $3 / 2$ to prevent aliasing of nonlinear terms. This corresponds to $N_{x}=2400$ spatial points with spacing $\Delta x=7.7 \times 10^{-5}$ to $7.9 \times 10^{-2}$ for an average spacing of $\Delta x=0.05$. The simulation runs from $t_{1}=0$ to $t_{1}=T=$ 10 , since the multiple-scale expansion of $\S 2.3$ is only accurate for times of $O(1 / \varepsilon)$. Adaptive time stepping is employed such that the Courant-Friedrichs-Lewy number is $(\Delta t) \max \left(\eta_{0}\right) /(\Delta x)=1$. For the unforced case, this corresponds to $\Delta t \approx 7.86 \times 10^{-3}$, increases to $1.04 \times 10^{-2}$ for $P=-0.25$ and decreases to $4.73 \times 10^{-4}$ for $P=0.25$. We found that linearly ramping up $P$ from 0 at $t_{1}=0$ to its full value at $t_{1}=\varepsilon$, or full, dimensional time $T_{0}=1 /\left(\sqrt{g h} k_{E}\right)$ (i.e. the time required to cross the inverse, effective wavenumber $1 / k_{E}$, or 'wave-crossing time') did not qualitatively modify the results, so we do not utilize such a ramp-up here. The spectral solver results in high numerical accuracy, with the normalized root-mean-square difference between the unforced $(P=0)$ profile $\eta_{0}$ at $t_{1}^{\prime}=10$ and the initial condition $\eta_{0}^{(0)}$ is $2 \times 10^{-13}$, and the normalized wave height change is $1-\left[\max \left(\eta_{0}\right)-\min \left(\eta_{0}\right)\right] /\left[\max \left(\eta_{0}^{(0)}\right)-\min \left(\eta_{0}^{(0)}\right)\right]=-1 \times 10^{-13}$.

We quantify the wave shape with the wave's energy $E$, skewness Sk and asymmetry As,

$$
E:=\left\langle\eta_{0}^{2}\right\rangle, \quad \mathrm{Sk}:=\frac{\left\langle\eta_{0}^{3}\right\rangle}{\left\langle\eta_{0}^{2}\right\rangle^{3 / 2}} \quad \text { and } \quad \mathrm{As}:=\frac{\left\langle\mathcal{H}\left\{\eta_{0}^{3}\right\}\right\rangle}{\left\langle\eta_{0}^{2}\right\rangle^{3 / 2}}, \quad \text { with }\langle f\rangle:=\frac{1}{L} \int_{-L / 2}^{L / 2} f \mathrm{~d} x \text {. }
$$

Here, $\mathcal{H}(f)$ is the Hilbert transform of $f$, defined as the imaginary part of $\mathcal{F}^{-1}(\mathcal{F}(f) 2 U)$ with $U$ the unit step function and $\mathcal{F}$ the Fourier transform. Since these definitions depend on the domain size $L$, we normalize the energy $E$ and skewness Sk by their initial values.

\section{Results}

We study the pressure magnitude's effect on solitary-wave evolution and shape by varying the KdV-Burgers equation's (2.18) one free parameter, $P k_{E} /\left(\rho_{w} g \varepsilon\right)$, with emphasis on the contrast between onshore $(P>0)$ and offshore wind $(P<0)$. We revert to denoting non-dimensional variables with primes and dimensional ones without.

The wave profile $\eta / h$ snapshots in figure 1 qualitatively show how the wave shape evolves over non-dimensional slow time $t_{1}^{\prime}=t \varepsilon \sqrt{g h} k_{E}$ in the unforced solitary wave's frame. The onshore wind generates wave growth, apparent at the wave crest (figure 1a), whereas the offshore wind causes decay (figure $1 b$ ). The wind also changes the phase speed, with the wave's acceleration (deceleration) under an onshore (offshore) wind visible by the advancing (receding) of the crest. This is expected due to the (unforced) solitary wave's nonlinear phase speed (2.20) dependence on the wave height $H$.

In shallow water, wave growth/decay and phase speed changes are well-known wind effects (e.g. Miles 1957; Cavaleri \& Rizzoli 1981), but wind-induced wave shape changes (Zdyrski \& Feddersen 2020) have not been previously studied for shallow-water systems. Such changes are visible in figure 1 where, despite the wave starting from a symmetric, solitary-wave initial condition, the wind induces a horizontal asymmetry in the wave shape, particularly on the rear face $(x<0)$ of the wave. The offshore wind (figure $1 b$ ) raises the rear base of the wave (near $x / h=-5$ ) relative to its initial profile (purple line), but the onshore wind (figure $1 a$ ) depresses the rear face and forms a small depression below the still water level at $t \varepsilon \sqrt{g h} k_{E}=5$ (blue line) which widens and deepens at $t \varepsilon \sqrt{g h} k_{E}=10$ (green line). Finally, the onshore wind (figure $1 a$ ) increases the maximum 


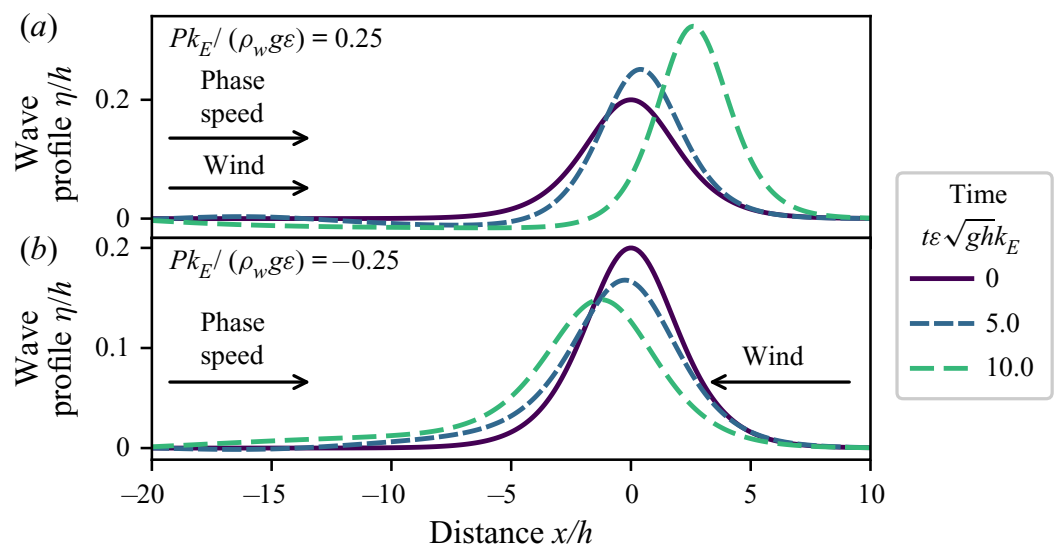

Figure 1. Solitary-wave evolution under $(a)$ onshore and $(b)$ offshore wind-induced surface pressure in the frame of the unforced solitary wave. Non-dimensional wave height $\eta / h$ versus non-dimensional distance $x / h$ for $\varepsilon=0.1, \mu_{E}=0.6,\left|P k_{E} /\left(\rho_{w} g \varepsilon\right)\right|=0.25$ and non-dimensional slow times $t_{1}^{\prime}=t \varepsilon \sqrt{g h} k_{E}=0,5$ and 10 , as indicated in the legend. Only a subset of the full spatial domain is shown. The arrows denote the wave propagation (phase speed) and wind direction.

wave-slope magnitude with time while the offshore wind (figure $1 b$ ) decreases it, although the windward side of the wave becomes steeper than the leeward side for both winds (up to $8 \%$ steeper for the time period shown). Although the equation is ill posed in the sense of Hadamard, the smooth solutions show that our solution is acceptable up to the current time and thus we are justified in neglecting a regularization scheme.

To further examine the wind-induced wave asymmetry, we fit $\eta$ to a reference solitary-wave profile $\eta_{\text {ref }}$ (2.19) by minimizing the $L_{1}$ difference, yielding the reference height $H_{r e f}\left(t_{1}\right)$ and peak location $x_{r e f}\left(t_{1}\right)$. The profile change is defined as $\Delta \eta(x):=$ $\eta-\eta_{\text {ref }}$ and is shown as a function of the reference wave-centred distance $\tilde{x}:=x-x_{\text {ref }}$ in figure 2. Notice that the profile change begins near the front face of the wave and has extrema for negative $\tilde{x}^{\prime}$ but with opposite signs for onshore and offshore winds. Additionally, the magnitude of the extrema decay with distance in the $-\tilde{x}$ direction. Finally, note that the onshore (offshore) wind generates a small peak (trough) at $\tilde{x}=0$ and two small troughs (peaks) near $\tilde{x} / h= \pm 3$, with the $\tilde{x}<0$ extrema larger than the $\tilde{x}>0$ one. This is analogous to a dispersive tail, well known in KdV-type systems (e.g. Hammack \& Segur 1974), and its appearance here helps explain the pressure-induced shape change (cf. §4.2).

The effect of wind on wave shape is quantified by the time evolution of wave shape statistics - energy, skewness and asymmetry - for onshore and offshore wind (figure 3 ). We plot all cases for initial steepness $\varepsilon=0.1$ up to slow time $t \varepsilon \sqrt{g h} k_{E}=10$, corresponding to $10 / \varepsilon=100$ wave-crossing times, $T_{0}=1 /\left(\sqrt{g h} k_{E}\right)$. The unforced case $(P=0)$ displays constant shape statistics and zero asymmetry, as expected. The normalized energy $E / E_{0}$ shows different growth/decay rates: the onshore wind $(P>0)$ causes accelerating wave growth while the offshore wind $(P<0)$ causes slowing wave decay (figure $3 a$ ). The energy of the unforced wave is virtually unchanged, with a normalized energy change of $1-E / E_{0}=-1 \times 10^{-13}$ at $t_{1}^{\prime}=10$. The onshore (offshore) wind causes the wave to become more (less) skewed over time, with the normalized skewness nearly symmetric about unity with respect to $\pm P$. Finally, the onshore wind causes a backwards tilt and negative asymmetry while the offshore wind increases the asymmetry and causes a forward tilt, which was also seen in figure 1. Notice that $\mid$ As $\mid$ is larger for onshore 


\section{T. Zdyrski and F. Feddersen}

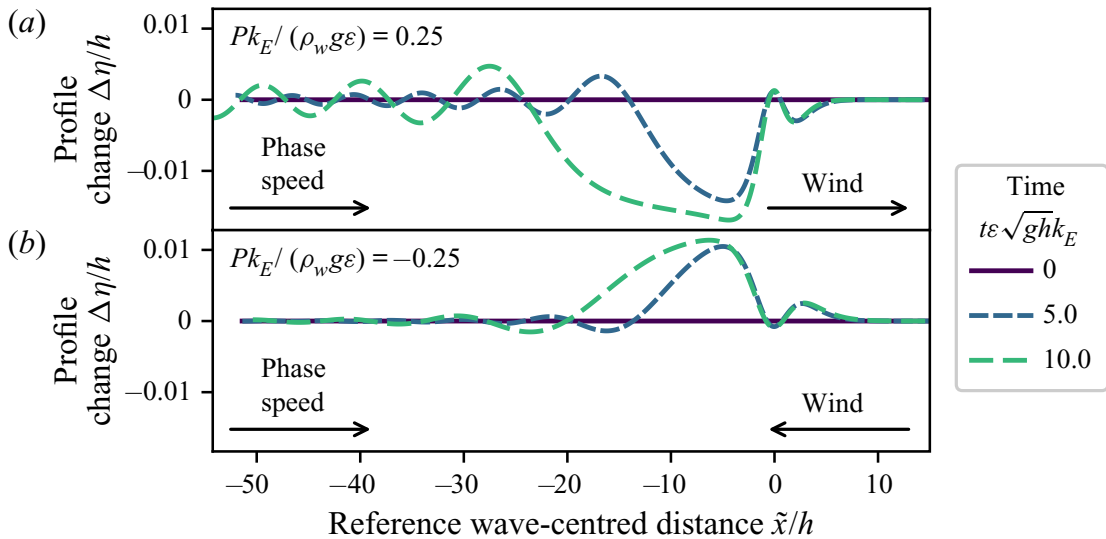

Figure 2. The non-dimensional profile change $\Delta \eta / h$ between the surface profile and reference solitary wave (2.19) under $(a)$ onshore and $(b)$ offshore Jeffreys forcing versus non-dimensional reference wave-centred distance $\tilde{x} / h$. Results are shown for $\varepsilon=0.1, \mu_{E}=0.6,\left|P k_{E} /\left(\rho_{w} g \varepsilon\right)\right|=0.25$ and non-dimensional slow times $t_{1}^{\prime}=t \varepsilon \sqrt{g h} k_{E}=0,5$ and 10, as indicated in the legend. Only a subset of the full spatial domain is shown. The arrows denote the direction of wave propagation (phase speed) or wind direction.

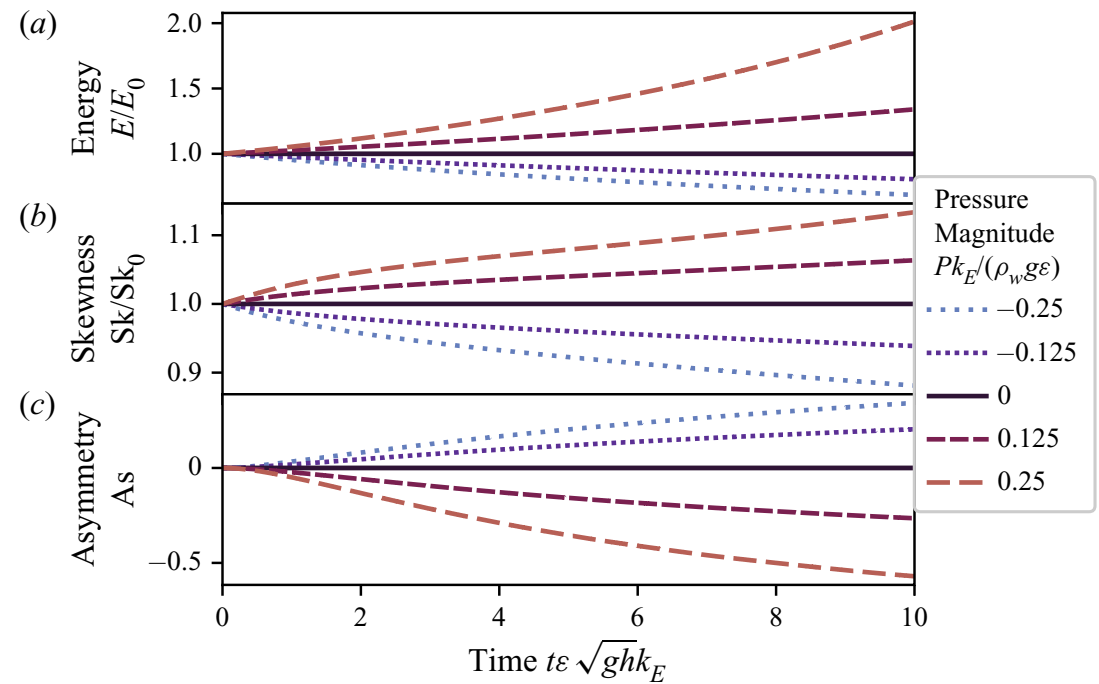

Figure 3. Solitary-wave shape statistics under onshore and offshore Jeffreys forcing versus non-dimensional slow time $t_{1}^{\prime}=t \varepsilon \sqrt{g h} k_{E}=0-10$. The $(a)$ energy (normalized by the initial energy), $(b)$ skewness (normalized by the initial skewness) and $(c)$ asymmetry are defined in $(2.21 a-c)$. Results are shown for $\varepsilon=0.1, \mu_{E}=0.6$ and pressure magnitude $\left|P k_{E} /\left(\rho_{w} g \varepsilon\right)\right|$ up to 0.25 , as indicated in the legend. The solid black line is the unforced case, $P=0$, and shows no growth or asymmetry and a constant skewness.

winds than offshore winds. Since the definitions of the skewness and asymmetry are insensitive to waveform scaling $\eta \rightarrow \lambda \eta$, this effect is not simply caused by the wave's growth/decay. Instead, the onshore wind generates a larger dispersive tail (figure 2), which is the asymmetric wave component. 


\section{Discussion}

\subsection{Wind speed estimation}

We now relate the non-dimensional pressure magnitude $P k_{E} /\left(\rho_{w} g\right)=O(\varepsilon)$ to the wind speed. First, we need a relationship between the surface pressure and wave energy $E$ $(2.21 a-c)$, which we can approximate using the standard procedure (e.g. Mei et al. 2005) of multiplying the (non-dimensional, denoted by primes) $\mathrm{KdV}-\mathrm{Burgers}$ equation (2.18) by $\eta_{0}^{\prime}$ and integrating from $x^{\prime}=-\infty$ to $\infty$ to obtain

$$
\frac{\partial}{\partial t_{1}^{\prime}} \int_{-\infty}^{\infty} \eta_{0}^{\prime 2} \mathrm{~d} x^{\prime}=\int_{-\infty}^{\infty} P^{\prime}\left(\frac{\partial \eta_{0}^{\prime}}{\partial x^{\prime}}\right)^{2} \mathrm{~d} x^{\prime} .
$$

The left integral is the non-dimensional energy $(2.21 a-c)$, so re-dimensionalizing and converting back to the full time $t$ gives the energy growth rate $\gamma$,

$$
\frac{\gamma}{c_{0} k_{E}}:=\frac{1}{c_{0} k_{E} E} \frac{\partial E}{\partial t}=\frac{P k_{E}}{\rho_{w} g} \frac{\left\langle\left(\partial_{x} \eta\right)^{2}\right\rangle}{\left\langle\left(k_{E} \eta\right)^{2}\right\rangle}=\frac{1}{5} \frac{P k_{E}}{\rho_{w} g},
$$

with $\left\langle\left(\partial_{x} \eta\right)^{2}\right\rangle /\left\langle\left(k_{E} \eta\right)^{2}\right\rangle=1 / 5$ evaluated with the initial, solitary-wave profile (2.19) and the linear, shallow-water phase speed $c_{0}=\sqrt{g h}$ coming from the re-dimensionalization of $t^{\prime}=t c_{0} k_{E}$ (2.7). Alternatively, a secondary multiple-scale approximation of the forward $\mathrm{KdV}$-Burgers equation has been used previously to derive the energy growth rate for solitary waves as (Zdyrski \& McGreevy 2019)

$$
E \propto \frac{1}{(1-\gamma t)^{2}} \quad \text { with } \gamma:=b\left[\frac{P k_{E}}{\rho_{w} g}\right] c_{0} k_{E},
$$

with analytically derived $b=2 / 15$. Numerically fitting (4.3) to our calculated energy instead yields $b=0.10081 \pm 0.00003$, similar to the analytic approximation. Note that the exponential energy growth (4.2) correctly approximates (4.3) for small times $\gamma t \ll 1$, and both expressions are consistent with the observed accelerating (decelerating) energy change for $P>0(P<0)$ in figure 3 .

Next, Jeffreys's (1925) theory relates the growth rate of periodic waves to the wind speed $U_{\lambda / 2}$, measured at a height equal to half the wavelength $z=\lambda / 2$, as

$$
\frac{\gamma}{c k}=S_{\lambda / 2} \frac{\rho_{a}}{\rho_{w}}\left(\frac{U_{\lambda / 2}}{c}-1\right)\left|\frac{U_{\lambda / 2}}{c}-1\right|,
$$

with $S_{\lambda / 2}$ a small, non-dimensional sheltering parameter potentially dependent on $\varepsilon$, $\mu_{E}$ and $U_{\lambda / 2} / c$. For simplicity, we approximate the nonlinear phase speed $c$ (given non-dimensionally in (2.20)) by its leading-order term $c_{0}=\sqrt{g h}$, yielding an error of only $10 \%$ in the subsequent calculations. Combining this approximation of (4.4) with (4.2) gives

$$
U_{\lambda / 2}=c_{0}\left(1 \pm \sqrt{\frac{1}{5}\left|\frac{P k_{E}}{\rho_{w} g}\right| \frac{\rho_{w}}{\rho_{a}} \frac{1}{S_{\lambda / 2}}}\right) .
$$

Here, the \pm corresponds to onshore $(+)$ or offshore $(-)$ winds. Note that changing the wind direction (i.e. \pm sign) while holding the surface pressure magnitude $\left|P k_{E} /\left(\rho_{w} g\right)\right|$ constant means onshore wind speeds $\left|U_{\lambda / 2}\right|$ will be larger than offshore wind speeds.

We can evaluate (4.5) for the parameters of $\S 3: \varepsilon=0.1, \mu_{E}=0.6$ and $P k /\left(\rho_{w} g \varepsilon\right)=$ 0.25 . Donelan et al. (2006) parameterized $S_{\lambda / 2}$ for periodic shallow-water waves with a 


\section{T. Zdyrski and F. Feddersen}

dependence on airflow separation: $S_{\lambda / 2}=4.91 \varepsilon \sqrt{\mu}$ for our non-separated flow (according to their criterion), with $\mu:=(k h)^{2}$. Assuming this holds approximately for solitary waves, we choose $\lambda=2 \pi / k_{E}=20 \mathrm{~m}$ to calculate the wind speed at $z=\lambda / 2=10 \mathrm{~m}$. This choice corresponds to a depth of $h=2.5 \mathrm{~m}$ and initial wave height $H_{0}=0.5 \mathrm{~m}$ and yields a wind speed of $U_{10}=22 \mathrm{~m} \mathrm{~s}^{-1}$, a physically realistic wind speed for strongly forced shallow-water waves. Weaker wind speeds will induce smaller surface pressures and thus take longer to change the wave shape.

\subsection{Physical mechanism of asymmetry generation}

Our initial, symmetric solitary waves (2.19) are permanent-form solutions of the unforced $\mathrm{KdV}$ equation. More generally, any initial solitary wave which does not exactly solve the $\mathrm{KdV}$ equation will evolve into a solitary wave and a trailing, dispersive tail according to the inverse scattering transform (e.g. Mei et al. 2005). In our system, the pressure continually perturbs the system away from the unforced $\mathrm{KdV}$ soliton solution resulting in a trailing, bound, dispersive tail (figure 2), which is responsible for the wave asymmetry. To see this, consider an initial, symmetric profile $\eta$. The pressure forcing term $P \partial_{x}^{2} \eta$ preserves the initial symmetry and induces a symmetric bound wave after a short time $\Delta t_{1}^{\prime} \ll 1$. This is apparent when considering the non-dimensional $\mathrm{KdV}$-Burgers equation (2.18) in the unforced solitary wave's frame (figure 1) at the initial time,

$$
\begin{gathered}
\left.\frac{\partial \eta_{0}^{\prime}}{\partial t_{1}}\right|_{t_{1}^{\prime}=0}=-P^{\prime} \frac{\partial^{2}}{\partial x^{2}}\left[\operatorname{sech}^{2}\left(\frac{x^{\prime}}{2}\right)\right] \\
\Longrightarrow \eta_{0}^{\prime}\left(x^{\prime}, \Delta t_{1}^{\prime}\right)=\left(2-P^{\prime} \Delta t_{1}^{\prime}\right) \operatorname{sech}^{2}\left(\frac{x^{\prime}}{2}\right)+P^{\prime} \Delta t_{1}^{\prime} \frac{3}{2} \operatorname{sech}^{4}\left(\frac{x^{\prime}}{2}\right) .
\end{gathered}
$$

The $P^{\prime} \Delta t_{1}^{\prime}$ terms generate a small bound wave with a peak (trough) at $x^{\prime}=0$ and troughs (peaks) symmetrically in front and behind the wave peak for onshore (offshore) wind. As time increases, the continual pressure forcing causes the bound wave to grow and lengthen behind the wave, as is apparent in figure 2 (e.g. $\tilde{x} / h=-20$ to 3 for $P^{\prime}=0.25$ and $t_{1}^{\prime}=10$ ).

The small numerical value $\left|P^{\prime}\right|=0.25 \ll 1$ used in $\S 3$ allows us to consider the wave's evolution as two steps with time scale separation. First, the pressure generates a bound wave (4.7) on the slow time scale, and then the wave evolves a dispersive tail on the fast time scale according to the inverse scattering transform of the unforced $\mathrm{KdV}$ equation. The dispersive tail in figure 2 (e.g. located left of $\tilde{x} / h=-20$ for $P^{\prime}=0.25$ and $t_{1}^{\prime}=10$ ) is analogous to the ubiquitous dispersive tails in prior studies on shallow-water solitary waves, such as figures $8(b)$ and $8(c)$ of Hammack \& Segur (1974). However, unlike dispersive tails generated from initial conditions which fail to satisfy the KdV equation, our tail is continually forced and lengthened by the wind forcing. Finally, interactions with the trailing, dispersive tail are responsible for lengthening the bound wave (4.7) behind, rather than ahead, of the solitary wave. Hence, the disturbance induced by the pressure forcing (4.7) has two effects on the wave. First, the wind slowly generates a bound wave which changes the height and width of the initial solitary wave, which is reflected in the growth (decay) and narrowing (widening) under onshore (offshore) winds in figure 1. Second, it quickly generates an asymmetric, dispersive tail behind the wave (figure 2), producing a greater shape change on the wave's rear face (figure 1). Finally, the different wind directions (i.e. pressure forcing signs) change the sign of the bound wave and dispersive tail and, hence, the sign of the asymmetry in figure 3. 


\subsection{Comparison to intermediate and deep water}

Zdyrski \& Feddersen (2020) investigated the effect of wind on Stokes-like waves in intermediate to deep water. This study, with wind coupled to waves in shallow water, finds qualitative agreement with those intermediate- and deep-water results. The shallow-water asymmetry magnitude increases as the pressure magnitude $P$ increases (figure 3 ), and figure 4(a) of Zdyrski \& Feddersen (2020) displayed a similar trend for the corresponding Jeffreys pressure profile, with positive (negative) pressure increasing (decreasing) the asymmetry. Although Zdyrski \& Feddersen (2020) compared their theoretical predictions to limited experimental results with $k h>1$, there are no appropriate experiments on wind-induced changes to wave shape in shallow water for comparison with our results. In addition to the Jeffreys pressure profile employed here, Zdyrski \& Feddersen (2020) also utilized a generalized Miles profile, only applicable to periodic waves, wherein the pressure was proportional to $\eta$ shifted by a distance parameter $\psi_{P} / k$. Future investigations could couple a higher-order Zakharov equation (e.g. Dommermuth \& Yue 1987) to a Jeffreys-type pressure forcing or to an atmospheric large eddy simulation, as was done for deep water by Hao \& Shen (2019). Although this analysis focuses on solitary waves, we also investigated the effect of wind on periodic waves using the cnoidal-wave $\mathrm{KdV}$ solutions as initial conditions. Wind-forced cnoidal waves displayed qualitatively similar shape changes with stronger onshore (offshore) wind causing the energy and skewness to increase (decrease) while the asymmetry decreased (increased) with time. Furthermore, results were qualitatively similar across multiple classes of cnoidal waves with different values of $\mu_{E}$, implying that these results apply rather generally.

\section{Conclusion}

Prior results (Zdyrski \& Feddersen 2020) in intermediate and deep water demonstrated that wind, acting through a wave-dependent surface pressure, can generate shape changes that become more pronounced in shallower water. Here, we produced a novel analysis of wind-induced wave shape changes in shallow water using a multiple-scale analysis to couple weak wind with small, shallow-water waves, i.e. $a_{0} / h \sim\left(k_{E} h\right)^{2} \sim P k /\left(\rho_{w} g\right) \ll 1$. This analysis produced a $\mathrm{KdV}$-Burgers equation governing the wave profile $\eta$, which we then solved numerically with a symmetric, solitary-wave initial condition. The deviations between the numerical results and a reference solitary wave had the form of a bound, dispersive tail, with differing signs for onshore and offshore wind. The tail's presence and shape are the result of a symmetric, pressure-induced shape change evolving under the inverse scattering transform. We also estimated the energy, skewness and asymmetry as functions of time and pressure magnitude. For onshore wind (positive $P$ ), the wave's energy and skewness increased with time while asymmetry decreased, while offshore wind produced the opposite effects. Furthermore, these effects were enhanced for strong pressures, and they reduced to the unforced case for $P=0$. The shape statistics found here show qualitative agreement with the results in intermediate and deep water. Finally, the wind speeds corresponding to these pressure differences were calculated and found to be physically realistic.

Acknowledgements. We are grateful to D.G. Grimes and M.S. Spydell for discussions on this work. Additionally, we thank the anonymous reviewers for their suggestions and comments.

Funding. We thank the National Science Foundation (OCE-1558695) and the Mark Walk Wolfinger Surfzone Processes Research Fund for their support of this work.

Declaration of interests. The authors report no conflict of interest. 


\section{T. Zdyrski and F. Feddersen}

\section{Author ORCIDs.}

(1) Thomas Zdyrski https://orcid.org/0000-0003-3039-172X;

(b) Falk Feddersen https://orcid.org/0000-0002-5488-9074.

\section{REFERENCES}

Ablowitz, M.J. 2011 Nonlinear Dispersive Waves: Asymptotic Analysis and Solitons, vol. 47. Cambridge University Press.

AntaR, N. \& DemiRay, H. 1999 Weakly nonlinear waves in a prestressed thin elastic tube containing a viscous fluid. Intl J. Engng Sci. 37 (14), 1859-1876.

Banner, M.L. \& Melville, W.K. 1976 On the separation of air flow over water waves. J. Fluid Mech. 77 (04), 825-842.

Burns, K.J., Vasil, G.M., Oishi, J.S., Leconnet, D. \& Brown, B.P. 2020 Dedalus: a flexible framework for numerical simulations with spectral methods. Phys. Rev. Res. 2, 023068.

CAVAleri, L. \& Rizzoli, P.M. 1981 Wind wave prediction in shallow water: theory and applications. J. Geophys. Res. 86 (C11), 10961-10973.

Dommermuth, D.G. \& Yue, D.K. 1987 A high-order spectral method for the study of nonlinear gravity waves. J. Fluid Mech. 184, 267-288.

Donelan, M.A., BABAnin, A.V., Young, I.R. \& BANNER, M.L. 2006 Wave-follower field measurements of the wind-input spectral function. Part II: parameterization of the wind input. J. Phys. Oceanogr. 36 (8), $1672-1689$.

Drake, T.G. \& Calantoni, J. 2001 Discrete particle model for sheet flow sediment transport in the nearshore. J. Geophys. Res. 106 (C9), 19859-19868.

Feddersen, F. \& Veron, F. 2005 Wind effects on shoaling wave shape. J. Phys. Oceanogr. 35 (7), $1223-1228$.

HADAMARD, J. 1902 Sur les problèmes aux dérivées partielles et leur signification physique. Princeton University Bulletin, pp. 49-52.

Hammack, J.L. \& SegUR, H. 1974 The Korteweg-de Vries equation and water waves. Part 2. Comparison with experiments. J. Fluid Mech. 65 (2), 289-314.

HAO, X. \& SHEN, L. 2019 Wind-wave coupling study using LES of wind and phase-resolved simulation of nonlinear waves. J. Fluid Mech. 874, 391-425.

HARA, T. \& Sullivan, P.P. 2015 Wave boundary layer turbulence over surface waves in a strongly forced condition. J. Phys. Oceanogr. 45 (3), 868-883.

HAYNE, G. 1980 Radar altimeter mean return waveforms from near-normal-incidence ocean surface scattering. IEEE Trans. Antennas Propag. 28 (5), 687-692.

Hoefel, F. \& ElgAR, S. 2003 Wave-induced sediment transport and sandbar migration. Science 299 (5614), $1885-1887$.

Husain, N.T., Hara, T., Buckley, M.P., Yousefi, K., Veron, F. \& Sullivan, P.P. 2019 Boundary layer turbulence over surface waves in a strongly forced condition: LES and observation. J. Phys. Oceanogr. 49 (8), 1997-2015.

JANSSEN, P.A.E.M. 1991 Quasi-linear theory of wind-wave generation applied to wave forecasting. J. Phys. Oceanogr. 21 (11), 1631-1642.

JefrReYs, H. 1925 On the formation of water waves by wind. Proc. R. Soc. Lond. A 107 (742), 189-206.

Johnson, R. 1972 Some numerical solutions of a variable-coefficient Korteweg-de Vries equation (with applications to solitary wave development on a shelf). J. Fluid Mech. 54 (1), 81-91.

Kharif, C., Giovanangeli, J.P., Touboul, J., Grare, L. \& Pelinovsky, E. 2008 Influence of wind on extreme wave events: experimental and numerical approaches. J. Fluid Mech. 594, 209-247.

KIVSHAR, Y.S. 1993 Dark solitons in nonlinear optics. IEEE J. Quant. Electron. 29 (1), 250-264.

Leykin, I.A., Donelan, M.A., Mellen, R.H. \& Mclaughlin, D.J. 1995 Asymmetry of wind waves studied in a laboratory tank. Nonlinear Process Geophys. 2 (3/4), 280-289.

LiN, P. 2004 A numerical study of solitary wave interaction with rectangular obstacles. Coast. Engng 51 (1), $35-51$.

Lin, P. \& LiU, P.L.-F. 1998 A numerical study of breaking waves in the surf zone. J. Fluid Mech. $359,239-264$.

LiU, Y., YANG, D., GuO, X. \& SHEN, L. 2010 Numerical study of pressure forcing of wind on dynamically evolving water waves. Phys. Fluids 22, 041704.

Mei, C.C., STIASSNIE, M. \& YUE, D.K.P. 2005 Theory and applications of ocean surface waves: nonlinear aspects. Advanced Series on Ocean Engineering, vol. 2. World Scientific.

MiLES, J.W. 1957 On the generation of surface waves by shear flows. J. Fluid Mech. 3 (2), 185-204.

913 A $27-12$ 


\section{Wind-induced wave shape changes in shallow water}

MiLES, J.W. 1979 On the Korteweg-de Vries equation for a gradually varying channel. J. Fluid Mech. 91 (1), $181-190$.

Monaghan, J.J. \& Kos, A. 1999 Solitary waves on a cretan beach. ASCE J. Waterway Port Coastal Ocean Engng 125 (3), 145-155.

MunK, W.H. 1949 The solitary wave theory and its application to surf problems. Ann. N.Y. Acad. Sci. 51 (3), 376-424.

Ono, H. 1972 Wave propagation in an inhomogeneous anharmonic lattice. J. Phys. Soc. Japan 32 (2), 332-336.

PHILlips, O.M. 1957 On the generation of waves by turbulent wind. J. Fluid Mech. 2 (5), 417-445.

SAHU, B. \& TRibeche, M. 2012 Nonextensive dust acoustic solitary and shock waves in nonplanar geometry. Astrophys. Space Sci. 338 (2), 259-264.

Sandstrom, H. \& OAKey, N. 1995 Dissipation in internal tides and solitary waves. J. Phys. Oceanogr. 25 (4), 604-614.

Soares, G.C., Fonseca, N. \& Pascoal, R. 2008 Abnormal wave-induced load effects in ship structures. J. Ship Res. 52 (1), 30-44.

Sullivan, P.P. \& MCWiLLiams, J.C. 2010 Dynamics of winds and currents coupled to surface waves. Annu. Rev. Fluid Mech. 42 (1), 19-42.

TIAN, Z. \& CHOI, W. 2013 Evolution of deep-water waves under wind forcing and wave breaking effects: numerical simulations and experimental assessment. Eur. J. Mech. 41, 11-22.

ToubOul, J. \& KhARIF, C. 2006 On the interaction of wind and extreme gravity waves due to modulational instability. Phys. Fluids 18, 108103.

Xu, G., Chen, Q. \& Chen, J. 2018 Prediction of solitary wave forces on coastal bridge decks using artificial neural networks. J. Bridge Engng 23, 04018023.

YAnG, Z., DenG, B.-Q. \& Shen, L. 2018 Direct numerical simulation of wind turbulence over breaking waves. J. Fluid Mech. 850, 120-155.

ZdYRSKI, T. \& Feddersen, F. 2020 Wind-induced changes to surface gravity wave shape in deep to intermediate water. J. Fluid Mech. 903, A31.

ZDYRSKi, T. \& MCGREEVY, J. 2019 Effects of dissipation on solitons in the hydrodynamic regime of graphene. Phys. Rev. B 99, 235435.

Zonta, F., Soldati, A. \& OnOrato, M. 2015 Growth and spectra of gravity-capillary waves in countercurrent air/water turbulent flow. J. Fluid Mech. 777, 245-259. 